\title{
DOCUMENTATION
}

\section{Compte rendu des essais contrôlés de chauffe-eau électriques à accumulation}

Sous le patronage des sociétés suivantes:

Société française des Electriciens,

Union des Syndicats de l'Electricité,

Syndicat professionnel des Producteurs et Distributeurs d'énergie électrique,

Comité national français de l'Eclairaye el du Chaulfage,

Office national des Recherches scientifiques et industrielles et des Inventions,

Société pour le développement des Applications de l'Electricilé $A P-E L$,

et avec le concours financier de cette dernière société, des essais contrôlés de chauffe-eau électriques à accumulation ont été faits tout récemment par le Laboratoire central d'Electricité.

Ces appareils, composés chacun, en principe, d'un réservoir cylindrique en tòle galvanisée, à la partie inférieure duquel se trouve un corps de chauffe constitué par une résistance électrique soigneusement isolée de l'eau a chauffer, sont mis en fonctionnement la nuit afin de bénéficier des tarifs réduits de vente de l'énergie qu'un grand nombre de compagnies de distribution d'électricité mettent à la disposition de leurs abonnés pendant les heures de nuit et les heures "creuses" de la journée.

Le courant est coupé automatiquement dès que la température désirée ou l'heure du changement de tarif est atteinte. L'eau chaude est, ensuite, utilisée, pendant le jour, selon les besoins

Aus essais en question, neuf constructeurs avaient présenté en tout 15 appareils répartis en trois séries de 30, 100, 200 litres.

Les essais exécutés ont prouvé que, d'une facon générale, les appareils essayés étaient conçus pour donner pleinement satisfaction à l'usager.

Les rendements ont, en effet, varié entre 89 et $92 \%$ pour les appareils hors pression, et entre 91 et $95 \%$ pour les appareils sous pression.

De plus, on peut dire que les pourcentages moyens d'eau chaude écuulée par rapport à la contenance de l'appareil avant que la température tombe de $5 \%$ ou $10 \%$ de la température maxima ont oscillé autour de :

$75 \%$ pour les petits appareils,

$85 \%$ pour les appareils moyens, et

$95 \%$ et mème au delà pour les gros appareils.

\section{Alliages à grande résistance mécanique susceptibles d'être employés en électro-technique}

Les métaux possédant de bonnes qualités mécaniques ne peuvent habituellement pas ètre employés en électrotechnique. M. M. G. Corson envisage, dans l'Electrical World, du 15 janvier, les traitements et mélanges susceptibles d'assurer à des métaux usuels (cuivre, aluminium, fer, chrome), à la foìs une conductivité électrique suffisante et de bonnes qualités mécaniques.

Depuis longtemps, des alliages ont rẻalisé ces qualités en assurant dans le réseau cristallin d'un composant de base, le remplacement de certains atomes de ce constituant par des atomes de l'élément d'alliage.

Quel que soit le mécanisme de la solution solide ainsi constituée, le résultat est presque toujours préjudiciable aux propriétés électriques primitives, et l'auteur cite de nombreux travaux qui meltent en valeur la difficulté de concilier les propriétés électriques et mécaniques des alliages. Le cas de duralumin est un de ceux qui illustrent le mieux le phénomène d'autant plus que, avec la mème solution solide primitive et un traitement thermique prolongé, effectué ultérieurement on constate la possibilité de revenir à la bonne conductibilité primitive : c'est ce qu'on a fait pour l'alliage appelé "aludur ", dont la résistivité est de 3,1 microhms, c'est-à-dire s'écartant beaucoup moins que celle du duralumin de la résistivité de l'aluminium pur.

Cet aludur s'obtient en associant à l'aluminium $1,2 \%$ de magnésium et $0,7 \%$ de silicium et en faisant subir, à la solution solide obtenue, un chauffage à $100^{\circ}$ durant une vingtaine d'heures. Le magnésium et le siliciun se trouvent précipités sous forme de particules colloïdales de siliciure de magnésium $\left(\mathrm{Mg}^{2} \mathrm{Si}\right)$, et avec la résistivité de 3,1 microhms l'alliage a une résistance d'environ $31 \mathrm{~kg} / \mathrm{mm}^{2}$, dépassant par conséquent de $250 \%$ celle de

\section{L'étalonnage des}

La Philadelphia Electric Company a installé à sa centrale de Richmond un dispositif d'étalonnage des compteurs d'eau utilisés pour les divers services de l'usine. Ce dispositif est décrit dans Power, du 8 mars, par M. Frederic G. Ely.

Le compteur ćtalon employé est du type à déversoir. Les ajutages des compteurs à calibrer sont placés dans des conduites d'essal spéciales intercalées dans la tuyauterie. Pour la détermination des indications de débit fournies par l'ajutage, on emploie deux instruments : un compteur semblable à celui normalement employé avec l'ajutage, et un manomètre différentiel à mercure et à tube incliné, qui permet de lire directement les dépressions en hauteur l'eau correspondantes. l'aluminium pur recuit. L'étirage à froid élève à $42 \mathrm{~kg} / \mathrm{mm}^{2}$ cette résistance, la rendant ainsi supérieure de $75 \%$ à celle de l'aluminium pur étiré à froid.

Les alliages de cuivre sont, l'après les travaux de l'auteur susceptibles d'ètre traités avantageusement suivant les mèmes principes, ainsi qu'il a été démontré par une longue étude méthodique d'alliages cuivre-nickel-silicium, cuivre-cobalt-silicium, cuivre-chrome-silicium et cuivre-nickel-bervllium.

Dans chaque addition d'éléments étrangers au cuivre, le traitement thermique qui suit la préparation de l'alliage ne laisse subsister en solution solide qu'une très minime proportion : par exemple $0,8 \%$ pour les éléments nickel et silicium dans les alliages cuivrenickel-silicium, $0,3 \%$ pour le cuivre et le silicium, $0,6 \%$ pour le nickel et le bervllium, $0,2 \%$ pour le chrome et le silicium.

Dans chaque cas, il semble que l'élément qui durcit le métal est partiellement constitué par cette petite proportion d'un composé tel que $\mathrm{Ni}^{2} \mathrm{Si}, \mathrm{Ni}^{2} \mathrm{Be}, \mathrm{Co}^{2} \mathrm{Si}, \mathrm{Cr}^{2} \mathrm{Si}$.

Les alliages cuivre-nickel-silicium, désignés sous le nom de "corsonites", peuvent servir pour les trolleys et les fils teléphoniques aériens. On peut aussi les employer à la constitution de pièces résistant à l'usure dans les machines et dispositifs électriques et mécaniques : anneaux collecteurs, sections de collecteurs, roues de contact, ressorts d'interrupteurs, etc.

En général. ces pièces s'usent très vite, les anneaux collecteurs encore plus que les sections de collecteurs segmentés, et il en est de même des roues de contact, qui doivent présenter à la fois des qualités électriques et des qualités mécaniques considérables. Le cobalt et le chrome promettent aussi d'importantes applications du mème ordre.

L.e Génie Givit.

Pour chaque valeur du débit, on fait un essai d'une clizaine de minutes. Pendant cet essai, on releve, toutes les 15 secondes, les indications fournies par le compteur et le manomètre différentiel. En opérant ainsi pour un certain nombre de valeurs du débit, on obtient une courbe de calibrage, de laquelle on déduit la courbe de correction du compteur. Au cours des essais, la température de l'cau est également observée et les corrections correspondantes sont faites.

I. but de ce calibrage n'est pas précisément dobtenir des mesures parfaites, mais plutot d'obtenir avec les differents compleurs de l'usine des mesures absolument comparables.

Le Ginie Civil. 


\section{Les laboratoires de l'Ecole technique de Hanovre}

Cette ecole vient ditre dotie de deux nowraux laboratoires : lun pour les appareils de levage et les pompes, l'autre pour les moteurs a combustion et l'etude technique de la chaleur.

Ces laboratoires sont décrits dans la zeitschrift des Vereines deutscher Ingenieure, du 29 janvier, par les professeurs klein et Neumann.

Lénergie est fournie an premier de ces laboratoires par la centrale de force motrice et de chauffe de l'école sous forme de vapeur ou de courant continu i 220 volts. Il existe onze moteurs électriques de 0.06 à $30 \mathrm{~kW}$, dont l'un de $25 \mathrm{~kW}$. De nombreux appareils servent à la mesure des forces, des vitesses, des débits, etc.. entre autres, un télétachymètre constitué par une petite dynamo dont l'induit entrainé par la machine étudiée tourne dans un champ homogène; la tension du courant produit, lue à distance sur un voltmètre, mesure le nombre de tours par minute. Ia différence entre deux vitesses, celle par exemple due au glissement dans les accouplements de freins, est mesurée par une méthode stroboscopique. Pour déterminer les rendements des diverses parties des engins de levage, il existe des balances construites de maniere a permettre la mesure du frottement des tourillons et des arbres dans les paliers.

La section des pompes comprend huit pompes antrifuges et une petite pompe à piston différentielle; une plate-forme cst réservée à l'essai des soupapes de pompes a piston.

Le second laboratoire comprend, comme moteurs à combustion, un moteur Diésel à deux cylindres et à quatre temps avec compresseur, un autre monocylindricue sans compresseur, un troisieme avec chambre de combustion séparée, enfin un semi-Diésel monocylindrique. Les moteurs à huiles légères sont représentés par deux moteurs à quatre temps, deux moteurs d'automobiles et un moteur marin à deux eylindres et à deux temps. Comme moteurs à ga\%, l'école dispose d'un moteur Deutz de 10 eh et d'un moteur monoevlindrique à quatre temps pouvant fonctionner, soit au gaz d'éclairage, soit au gaz pauve fourni par un gazogene brilant de l'anthracite ou du coke.

Pour l'étude de la chaleur. il existe un compresseur d'air ef une installation destinée à la mesure des coefficients de transmission de la chaleur.

le Ginie Civil.

\section{Le travail continu dans les fonderies américaines}

MI. C. Pardun et R. Agte rendent compte, dans le Stahl und Eisen, du 23 décembre, des observations qu'ils ont pu faire, au cours d'un récent vovage sur le fonctionnement des fonderies américaines, en ce qui concerne la fabrication des pièces de grancle série. Le système est basé en principe sur une circulation des chàssis suivant un circuit fermé qui les amène successivement aux machines à mouler, puis aux poches de coulée; ensuite, après un trajet correspondant à un temps de refroidissement suffisant. les pièces arrivent au démoulage, d'où les chàssis repartent pour une nonvelle opération, tandis que les pièces vont subir leur ébarbage, leur usinage, ete, et que le sable est envoyé aux appareils de préparation; de là, apres traitement convenable, il revient aux machines à mouler. Les auteurs montrent, a l'aide d'exemples, les dispositions les plus convenables dans les différents cas.

L. Gónie Civil.

\section{Le flux supplémentaire monophasé des transformateurs triphasés}

La charge d'un transformateur triphasé, quelle qu'en soit la nature, est généralement dissymétrique. Dans une étude que publie l'Elektrotechnik und Maschinenbau, du 30 janvier, M. Widmar, professeur à l'Université de Ljubljana (Yougoslavie), montre que cette dissymétrie a pour conséquence la production d'un flux supplémentaire monophasé, susceptible de provoquer des troubles importants dans l'exploitation. L'auteur étudie d'abord les causes donnant naissance à ce flux qui, en dehors de la dissrmétrie de la charge, résident dans l'inégalité des trois courants magnétisants. Le flux monophasé est encore plus intense quand le transformateur est couplé en triangle, que dans le cas du couplage en étoile.

L'auteur étudie ensuite les effets du flux monophasé. Celui-ci induit dans les enroulements des tensions qui se composent avec les tensions étoilées; il en résulte un déplacement du triangle de ces tensions et du point neutre du système; les tensions sont modifiées non seulement en position, mais aussi en grandeur: le flux supplémentaire se traduit pratiquement par des chutes de tension très fâcheuses.

L'auteur signale un autre phénomène provoqué par le flux monophasé : la variation de la densité des lignes de force dans le fer, puis il passe à l'examen dans quelques cas particuliers, des inconvénients du flux monophasé.

Quand une seule des phases d'un transformateur triphasé

\section{La protection contre l'humidité des}

Les petits transformateurs des réseaux ruraux sont souvent victimes d'accidents dus à leur protection insuffisante, notamment contre l'humidité atmosphérique qui, à la longue, vient à bout des meilleurs isolements. Il suffit de $1 / 1$ ooo d'eau dans une huile pour diminuer de moitié son pouvoir isolant.

M. Lapiné, chef de service aux Ateliers de Constructions électriques de Lyon et du Dauphiné, déerit, dans la Revue générale de l'Electricité du 26 février, des dispositifs de construction très est chargée, la tension supplémentaire atteint $10 \%$ de la tension etoilée dans le cas du couplage en étoile sans fil neutre an primaire, et $20 \%$ dans le cas du couplage en triangle.

On peut négliger les flux dus aux courants magnetisants, tant que la charge est symétrique, mais il est nécessaire d'étouffer le flux monophasé quand cette srmétrie n'existe plus: l'anteur indique comme moyen d'obtenir ce résultat l'emploi d'une spire en court-circuit entourant le flux supplémentaire. Il montre la nécessité de réduire le plus possible la résistance ohmique de cette spire pour en augmenter l'efficacité, et préconise son utilisation pour étouffer le flux monophasé de l'enroulement primaire du transformateur en le connectant en triangle au lieu de le coupler en étoile. Cette solution donne des résultats l'autant plus satisfaisants qu'il s'agit d'un transformateur de plus forte puissance, et présente l'avantage, en cas d'accident sur wne phase, de permettre de continuer l'exploitation avec le couplage en triangle.

M. Widmar signale encore un autre phénomène important la cuve des transformateurs constitue une spire en court-circuit et offre un passage commode aux lignes de force du flux monophasé. Il en résulte des pertes, qu'il est d'ailleurs facile de mettre en évidence en essayant le transformateur, d'abord dans sa cuve, puis après len avoir sorti.

Le Génie Civil.

\section{transformateurs de réseaux ruraux}

simples, destinćs à empècher l'introduction de l'humidité daus l'intérieur du transformateur, notamment le "réservoir d'expansion "et l' "assécheur d'air " à chlorure de calcium; il indirfue également des modes de construction évitant le démontage du couvercle pour le changement des prises de courant, démontage qui donne lieu, trop souvent, à des défectuosités d'étanchéilé, après remontage, cette opération étant faite dans des conditions incommodes.

\section{La nouvelle sous-station automatique, de Bruxelles, à redresseurs à vapeur de mercure}

Récemment fournie à la ville de Bruxelles, et équipée par la Brown-Bovery $\mathrm{C}^{\circ}$, cette sous-station alimente aujourd'hui une grande partie du réseau de la capitale belge (ćclairage et force) et comprend 4 groupes de $400 \mathrm{KW}$. recevant le primaire, triphasé, a) $5.250 \mathrm{~V}$. et se somposant, chacun, de 2 redresseurs gu'alimente un transfo.

Le courant redressé et ahaissé est ensuite envoyé dans le réseau général de distribution à $220 \mathrm{~V}, \cdots-3$ conducteurs. - - (Un groupe de $600 \mathrm{KW}$. équipe pareille sous-station à Ostende.

Rev, B. B. C., 11-1926. 


\section{L'électrification rurale en Suède}

En Suede, la production et la distribution de l'énergie électrique sont entre les mains de deux organismes principaux : l'Etat, qui dessert la Suèce centrale, et une Société privée, résultant de la fusion de plusieurs réseaux, qui alimente la partie méridionale du pays. Ces deux réseaux sont alimentés principalement par les isines hydro-electricues et possèdent également un certain nombre de centrales thermiques de secours.

Les grands centres producteurs sont reliés actuellement par des lignes à 130.000 volts, qui doivent être portées ultérieurement à 220.000 volts.

Les lignes principales de distribution fonctionnent a la tension de 50.000 volts el les lignes rurales, dont la longueur moyenne varie de 200 a $300 \mathrm{~km}$, distribuent le courant à la tension de 20.000 volts.

Le courant est livré aux agriculteurs soit à 1.500 soit à 39000 volts; pour cela, des postes transformateurs sont établis aux points d'utilisation. Ces postes sont, soit du type intéricur, soit du type cxtéricur, soit enfin du type sur poteau. Ces derniers postes sont établis d'une façon intéressante : les trois fusibles haute tension placés sur la dérivation alimentant le transformateur sont montés sur un calre mobile tournant autour d'un axe horizontal et rabattable juscuu'à hauteur d'homme. Ce dispositif permet d'éviter de monter sur le pylòne pour remplacer les fusibles, et diminue les dangers d'électrocution.

M. Ekstrom projette un film sur l'utilisation de l'électricité dans la ferme suédoise. Une ferme doit posséder trois types de moteurs électriques :

1. Des moteurs de 1 a 2 ch pour le service des étables et des ccuries;

20 Des moteurs de 3 a 5 ch pour les laiteries, manutentions et travaux divers;

$3^{\circ}$ Des gros moteurs de plus de 5 ch p i r la batteuse.

D'une façon générale, les petits moteurs sont du type portatif ; les antres sont, soit du type portatif, soit du type fixe.

Dans l'étable, le moteur électrique est utilisé pour traire mécaniquement les vaches, pour distribuer la nourriture aux animaux. pour assurer le service d'eau, tant pour la boisson du bétail que pour les soins de propreté.

Dans les laiteries, le moteur électrique, soit par commande individuelle, soit par commande générale, actionne les écrémeuses, les barattes, les malaxeurs, les machines à laver, etc.

En Suede, ces divers moteurs sont construits pour fonctionner a la tension de 380 volts; dans les endroits où on n'a pas de prise pour cette tension, on utilise des transformateurs portatifs que l'on branche sur les lignes à 3.000 ou à 1.500 volts. Lorsque la distribution est faite à cette dernière tension, il arrive souvent (que des moteurs de forte puissance soient branchés directement sur le circuit.

Quand on veut utiliser un moteur électrique en plein champ, on dispose d'un matériel amovible qui permet de poser rapidement une ligne de haute tension.

En Suede, la manutention électrique a pris un large développement dans les travaux agricoles. Avant de charger les fourragères, les agriculteurs disposent dans le fond de celles-ci un systeme très simple de cordes qui sert à maintenir le chargement et permet, une fois la voiture arrivée à la ferme, de la décharger en une seule fois a l'aide d'un palan électrique. Cette façon de procéder conduit à des économies de main-d'cuvre très importantes et a eu pour conséquence de modifier profondément la construction des bâtiments des fermes suédoises. Autrefois, la ferme se composait de deux bâtiments principaux : l'étable surmontée d'un petit grenier et une grange contiguë. Quand l'approvisionnement du grenier était epuisé, il fallait puiser dans la grange pour le reconstituer. Maintenant, grâce aux facilités de manutention données par l'électricité, le grenier a disparu et a été remplacé par une grange construite directement au-dessus des étables; un palan électrique dessert cette grange et permet une manutention facile et rapide du fourrage. Cette nouvelle disposition procure, en plus des économies de main-d'ouvre, une importante économie dans la construction des batiments.

Les ventilateurs électriques ont trouvé également de larges applications dans les fermes suédoises et sont utilisés pour chasser la vamure, aspirer la paille et monter les grains dans les greniers à blé.

L'électricité est encore utilisée par les maraîchers pour le chauffage des châssis vitrés. Des essais méthodiques faits sur 60.000 pieds de salade ont permis de réduire de plus de $25 \%$ le temps de végétation; l'installation de chauffage est effectuée de la facon suivante : des câbles alimentés à la tension de 200 volts sont placés dans des tubes au fond des bàches vitrées et recouverts par la terre végétale dans laquelle on fait la plantation.

Les résultats obtenus en Suède par l'emploi de l'électricité dans les travaux agricoles ont été si satisfaisants quactuellement plusieurs milliers de fermes sont électrifiées dans ce pays.

M. Ekstrom émet le voru que la France suive l'exemple donné par la Suède pour le plus grand bien de son agriculture et invite les agriculteurs et les électriciens français à venir visiter les installations agricoles modèles de son pays. Ses compatriotes et lui mème se feront un plaisir de leur donner tous les renseignements qu'ils pourraient désirer et de les faire profiter de l'expérience qu'ils ont acquise.

Avant de terminer, M. Ekstrom signale que l'électricité rend les plus grands services pour la cuisson des aliments. Il vient d'ètre inventé en Suède un fourneau électrique à accumulation qui permet d'utiliser le courant d'une façon rationnelle et peut subir de longues interruptions de courant sans dommage pour la cuisson des aliments qu'il renferme. Cette propriété permet aux usagers de profiter de tarifs spéciaux, à la condition qu'ils mettent leurs fourneaux hors circuit pendant les heures de pointe des réseaux.

En principe, le fourneau se compose d'un bloc de fonte qu'un premier enroulement chauffe d'une façon continue à une température voisine de $500^{\circ}$. Si la temperature tombe au-dessous de $500^{\circ}$, un thermostat met en circuit un deuxième enroulement de chauffage jusqu'au moment où la température est remont é à la valeur voulue.

l.e fourneau est complété par une plaque de cuisson, un four dans lequel la température est maintenue à $90^{\circ} \mathrm{C}$, et un réchauffeur d'eau qui se met en action quand la température dépasse $500^{\circ} \mathrm{C}$.

Les résultats obtenus par ce four sont excellents, tant au point de vue de la cuisson que par l'économie importante du temps qu'il procure.

La question de la cuisson électrique a été également étudiée de très près en Norvège et les résultats obtenus dans ce pays sont tout a fait remarquables.

\section{La part de l'éclairage électrique dans le débit des stations centrales}

Le courant fourni par les centrales électriques fut d'abord utilisé presque exclusivement à l'éclairage: puis il servit à obtenir la force notrice et ce fut cette application que les centrales cherchèrent surtout à développer. Pour justifier cette tendence, on invoqua les difficultés du problème de l'éclairage et on mit en doute les résultats économiques que procure la fourniture du courant pour la lumière. M. A. G. Arnold a fait sur ce sujet, devant les représentants des centrales d'électricité, une conférence que reproduit l'Elektrotechnik und Maschinenbau, du 23 janvier. Deux graphiques montrent: l'un, l'état actuel de l'électrification aux Etats-Unis et ce que devrait etre cette électrification, en faisant ressortir la part considérable que devrait prendre l'éclairage par rapport aux autres applications de l'électricité ; l'autre graphique montre la variation du rapport en Allemagne des kilowattslumière aux kilowatts-force dans la période déjà ancienne de 1888 à 1908 .

I.autcur étudie ensuite les facteurs sur lesquels il est poss le d'agir pour augmenter le revenu brut des centrales d'électricité : augmentation de la consommation, tarifs, clientèle, normalisation des tensions, réduction des pertes thermiques et électriques, diminution du prix de la construction de la centrale et des installations de distribution, jonction de plusieurs centrales, augmentation de la durée d'utilisation.

Pour augmenter ce revenu des centrales, les efforts séparés de chacune d'elles ne sauraient suffire: il est indispensable de coordonner les efforts de tous. Les-résultats ainsi obtenus en Amérique, où $67 \%$ des recettes proviennent de l'utilisation du courant à la lumière et aux usages domestiques, montrent la voie à suivre pour les autres pars. Certains l'ont déjà compris. L'auteur conclut en montrant la nécessité d'ume organisation centrale embrassant tous les cercles intéressés à l'industrie électrique et aux industries voisines, ainsi que les administrations et les instituts; cette organisation existe en Allemagne : c'est la "Société allemande pour l'éclairage ".
Le Génie Civil. 


\section{Les intensités admissibles dans les conducteurs isolés}

Les prescriptions actuelles d'installations de l'Association des Electriciens allemands concernant les intensités admissibles dans les conducteurs isolés sont déduites de mesures d'essais faits en 1907, et basées notamment sur une température limite de $50^{\circ}$ pour la couche isolante de caoutchouc. Depuis ce temps, le développement des appareils électriques domestiques de toutes sortes a entrainé une augmentation considérable des intensités passant dans les conducteurs usuels, et il a été jugé nécessaire de procéder à de nouveaux essais sur les intensités admissibles dans les conducteurs de faible section, afin de modifier éventuellement les prescriptions actuelles.

Les mesures, dont M. Apt donne les résultats dans l'Elektrotechnische Zeitschrift du 20 janvier, ont porté d'une part sur les ćchauffements des fils isolés de différentes sortes et de sections comprise's entre $0^{\mathrm{mm} 2} 5$ et $2^{\mathrm{mm} 2} 5$, et d'autre part sur les propriétés de la couche isolante de caoutchouc à différentes températures; il en résulte qu'un échauffement prolongé à $50^{\circ}$ et $60^{\circ}$ n'entraìne aucune modification des propriétés mécaniques, résistance et élasticité, de la couche isolante. A 700 . l'échauffement prolongé diminue la résistance sans altérer l'élasticité. Avec du caoutchouc de composi- tion non réglementaire, leur résistance est détruite après échauffement a $70^{\circ}$, de telle sorte qu'atcune modification des prescriptions sur la composition des isolants de caoutchoue ne doit etre envisagée.

En conclusion de ces mesures, il est possible, par suite de l'ćlévation a $60^{\circ}$ de la temperature limite admissible pour la couche de caoutchouc, dadmettre dans les conducteurs isolés des intensités de courant sensiblement plus elevées que celles prescrites actuellement. L'intensité permanente maximum peut ainsi être portée de 7,5 à 10 ampères pour les fils isolés de $0^{\mathrm{mm} z} 5$, et a 12 ampères pour cenx de $0 \mathrm{~mm}$ m5. En fait, l'intensité admissible dépend beaucoup du dispositif de protection employé : les coupecircuits fusibles sont peu favorables, parce que leur ichauffement se produit dans des conditions très différentes du conducteur lui-mème. Lappareil idéal serait celui qui protégerait le conducteur, non contre les surintensités, mais contre les échaufements dangereux, c'est-à-dire qui posséderait les mèmes caractéristiques d'échauffement que celui-ci; on peut esperer que les recherches entreprises aboutiront dans cette voie.

Le Génie Givil.

\section{Accouplements automatiques permettant le démarrage à pleine charge des moteurs asynchrones en court-circuit}

Le but de ces accouplements, décrits par M. Obermoser, dans l'Elektrotechnische Zeilschrifl, du 13 janvier, est de permettre l'emploi des moteurs asynchrones à cage d'écureull démarrant sous charge, en demeurant dans les limites prescrites notamment par les règlements de l'Association des Electriciens allemands concernant l'à-coup de courant au démarrage. En raison des nombreux avantages des moteurs à induit en court-circuit, par rapport aux moteurs à bagues, on a cherché depuis longtemps. pour en rendre l'emploi admissible avec des puissances assez élevées, des dispositifs remédiant à leurs mauvaises caractéristiques de démarrage.

Telles sont les poulies dites centrifuges, comportant des masses centrifuges rappelées par des ressorts et venant assurer pour une certaine vitesse l'entraînement de la partie folle de la poulie de façon à réaliser le démarrage à vide du moteur. Bien que l'à-coup de courant au démarrage soit notablement réduit, l'examen des oscillogrammes montre que ce courant dépasse encore sensiblement les limites prescrites, et que le problème ne peut être considéré comme résolu. Le résultat est analogue avec les poulies basées sur l'essorage centrifuge de l'huile graissant les surfaces de frottement de la partie fixe et de la partie folle, et dans lesquelles l'embrayage résulte de l'accroissement important du coefficient de frottement consécutif à cet essorage.

Pour résoudre complètement le problème, il est d'abord nécessaire d'alimenter, au démarrage, le moteur sous tension réduite, de façon à limiter l'à-coup de courant instantané qui serait sans cela inadmissible, même en démarrant le moteur à vide. Pratiquement, on utilise à cet effet les commutateurs ćtoile-triangle. L'embrayage proprement dit ne doit s'effectuer qu'après la commutation sous pleine tension. Sur ces données, différentes solutions satisfaisantes en principe peuvent être envisagées : en premier lieu, le dispositil d'embrayage peut être prévu de façon à transmettre pendant le démarrage en étoile un couple réduit, mais suffisant pour que le passage au triangle s'accompagne d'une accólération brusque, utilisable pour déclencher le dispositif d'embrayage définitif. On peut, également munir l'accouplement d'un système à retardement tel que l'embrayage ne s'effectue qu'après un laps de temps supérieur à celui nécessaire pour la manouvre du commutateur étoile-triangle.

L'accouplement "Albo " est basé sur le fait que le rotor d'un moteur asynchrone tournant à vide subit un ralentissement brusque au moment de la commutation étoile-triangle. Ce ralentissement est utilisé pour déclencher l'embrayage. Le fonctionnement de l'accouplement, qui ne comporte que des pièces mécaniques, est ainsi directement provoqué par la manœuvre du commutateur du moteur. L'examen des oscillogrammes montre que tout à-coup de courant exagéré pendant le démarrage est supprimé et que le problème du démarrage des moteurs en court-circuit, quelle que soit leur puissance, peut être considéré comme résolu.

\section{Ie Génie Civil.}

\section{L'état actuel de la construction des machines agricoles}

En j.in 1926 s'est tenue à Breslau l'exposition annuelle de la Société allemande d'agriculture. MM. Erhardt et Gerdes donnent une description des principales machines exposées, dans la Zeitschrift des Vereines deutscher Ingenieure du 8 janvier.

Parmi les machines motrices, ils signalent notamment une locomotive à vapeur Wolf de $33 \mathrm{ch}$, puissance continue, pesant seulement $2.300 \mathrm{~kg}$, grâce à une disposition nouvelle; des petites turbines hydrauliques de puissance inférieure à $3 \mathrm{~kW}$ avec générateurs de courant électrique montés en porte à faux; des tracteurs avec moteurs Diesel et semi-Diesel, en particulier un tracteur dont les roues arrières sont des cylindres de $1^{\mathrm{m}} 66$ de diamètre, d'une largeur totale de $2 \mathrm{~m} 70$, son poids est de $6.300 \mathrm{~kg}$; des tracteurs à chenilles de différents modèles, dont un pouvant fonctionner à volonté comme tracteur à roues ou à chenilles.

Les auteurs passent ensuite en revue les charrues pour tracteurs (charrues-bascules et charrues réversibles), les semoirs et les cultivateurs, les faucheuses, les moissonneuses, les machines a battre et les transporteurs-élévateurs.

Ils signalent aussi quelques machines accessoires, pompes à purin, ume rampe permettant de faire monter les wagonmets des voies étroites sur une plate-forme d'où il est facile de faire basculer leur contenu dans les wagons à voie normale. Ils examinent enfin différentes pièces détachées, d'une importance particulière pour la construction des machines agricoles. Le Génie Civil.

\section{Le dégrillage à l'usine de Rheinfelden}

Cette opération, particulièrement importante, sur 135 mètres de longueur de grilles de 8 mètres de hauteur, s'effectuait autrefois à la main au moyen de râteaux à long manche. Après un essai de dégrillage mécanique demeuré sans grand succès, on vient d'installer à l'usine de Rheinfelden une machine à dégriller du système Jonneret, construite à Genève, dans les ateliers de même nom. Cette machine est remarquablement décrite et représentée illustrativement dans l'étude de MM. Haas et Bitterli - trop longue pour être analyséc ici … à laquelle nous renverrons nos lecteurs qui seraient particulièrement intéressés.

Zeitsch des Vereines deutscher Ing, 6-11.

J. B. 


\section{Les résultats de l'électrification des Chemins de fer du Nord de l'Espagne}

Daprès une décision prise en 1923, la Compania de los Caminos de Hicro del Norte de Espana a réalisé l'electrification d'une partie très aceidentée de son réseau. Cette électrification s'est faite en 1921 , et les résultats de l'exercice 1925 permettent déja d'en apprécicr les conséquences.

Wans la Ciemeral Electric Review, de décembre, M. W. D. Bearce expose ces résultats, en supposant connues les installations réalisées pour l'électrification du réseau, c'est-à-dire pour la substitution (le 12 locomotives électriques à 30 machines a vapeur pour assurer le tratic sur des lignes d'une longueur de $100 \mathrm{~km}$. environ.

I. a puissance totale des locomotives à vapeur s'élevait à $29.500 \mathrm{ch}$, celle du matériel électrique de remplacement, à une puissanec totale continuc de $18.960 \mathrm{ch}$. Bien que cette puissance continue soit plus faible que celle des locomotives à vapeur, la capacite te surcharge des équipements permet d'assurer sans difficulte le mêne service, en réalisant une economie s'élevant à $50 \%$ sur le prix de l'énergie nécessaire.
Le nombre des locomotives électriques étant plus réduit, leur activité et la suractivité possibles du matériel électrique constituent un avantage que l'expérience met bien en évidence. Les déplacements inutiles, les retours aux dépôts, etc., représentent de nombreux kilomètres inutilement parcourus par les machines à vapeur et leur tender, et on constate, sur les parcours qu'on avait relevés lors de l'exploitation à vapeur, une économie de $47 \%$ de tracteurs-kilomètres depuis l'abandon de la locomotive à vapeur. Comme celle-ci se double toujours d'un tender, les tonnes kilometres totales afférentes aux tracteurs se trouvent réduites de $83 \%$ par l'électrification.

Le coût des réparations et de l'entretien est lui-mème réduit de $72 \%$, celui des équipes d'exploitation de $63 \%$. Le prix du remorquage des trains se trouve réduit de $31 \%$. Cette économie ne représente pas moins de 1.193 .400 pesetas pour le premier exercice annuel depuis lélectrification.

Ie Génie Civil

\section{La réduction des pertes parasites aux extrémités du stator des alternateurs à grand pas solaire}

Tous les constructeurs connaissent l'importance des pertes parasites clans les tites de bobines et pieces d'extrémités des stators d'alternateurs à grand pas polaire, surtout quand il s'agit d'unités de fortes puissances. Cette question a été rarcment traitie lans la presse techniçue, surtout dans la presse française; aussi crovons-nous devoir signaler un article de $M$. de Pistoye, paru dans la Revue générale de l'Electricilé, du 5 février, qui la traite 'n détail, en indiquant les mémoires publiés à l'étranger sur ce slijet.

Les pertes dans les conducteurs massifs logés dans les encoches du stator sont importantes si lon ne prend pas une précaution qui, d'ailleurs, est usitce depuis longtemps, celle de croiser les conducteurs élémentaires mis en parallèle.

D'autres sources de pertes parasites sont localisées dans les tètes de bobines d'induit, et à leur voisinage. Elles ont été étudiées, en Allemagne, par Pohl et par Rurlemberg, mais leur détermination theorique exacte est presque impossible.

Enfin, on constate d'autres pertes par les boulons, cercles et plaques en acier, servant à fixer les tètes de bobines, ainsi que par les joues de serrage des tôles d'induit.

Certains modes de construction, qu'indique l'auteur, concourent à limiter ces diverses pertes: notamment, l'emploi de bpbinages à pas raccourci, et surtout l'emploi d'un métal non magnétiqua pour constituer les joues de serrage des tôles d'induit et les protecteurs de bobinages. Le métal qui convient le mieux, tan au point de vue électrique qu'à celui de l'économie, est une fonte au nickel de fabrication anglaise, dite fonte "nomag ", étudiée par les Etablissements Ferranti. Le Génie Civil.

\section{L'électrification rurale dans la région parisienne}

Des communications ayant déjà été présentées à la Société sur le labourage électrique, M. Bitouzet a limité son sujet aux résultats donnés par le labourage dans la région parisienne.

La "Société électrique de travaux agricoles ", qui y fait le nétier d'entrepreneur de labourage électricue, utilise pour cela trois types de matériel

Le type de la Société générale agricole, qui comprend deux trémies de 125 ch chacun; le type de la Compagnie d'entreprises électromécaniques, qui n'emploie qu'un seul treuil de $60 \mathrm{ch}$ accompagné des poulies de renvoi nécessaires, et le type Estrade ou Compagnie méridionale, qui possède deux treuils de 40 à $60 \mathrm{ch}$.

Avec ce matériel, il a été labouré dans la région parisienne pendant la dernière campagne 1.500 hectares à une profondeur de 30 à $35 \mathrm{~cm}$. La Société compte augmenter la surface labourée en exécutant du travail de nuit, malgré la difficulté que présente alors l'éclairage des chantiers.

Le labourage électrique, gràce à la souplesse de son moteur, a montré qu'il triomphe beaucoup plus facilement que les autres modes de labourage, des obstacles habituellement rencontrés, comme les racines et les masses glaiseuses.

L.a plus grande difficulté est le recrutement du personnel : les clectriciens et mécaniciens de profession supportant difficilement de travailler l'hiver à la campagne, il a fallu recourir à la maind'œuvre agricole ordinaire, mais cette main-d'œuvre n'est pas apte à exécuter les réparations courantes. En général, il faut essayer cinq hommes sur un chantier pour en trouver un qui convienne. Les meilleurs résultats sont obtenus avec des ouvriers d'origine agricole ayant passé deux ou trois ans dans une usine éloignéc des grands centres industriels.

La clientèle s'est montrée très satisfaite et a trouvé qu'aucun autre mode de labourage n'avait procuré une telle perfection dans le travail.

M. Bitouzet fit ensuite passer un film montrant les chantiers de labourage électrique, et conclut que, pour que l'agriculture francaise reprenne la place qu'elle occupait jadis, il est nécessaire qu'elle améliore son outillage en recourant dans la plus large mesure au courant électrique : il est donc indispensable de développer largement les réseaux ruraux.

L'Etat et les électriciens ont fait des efforts considérables dans ce sens, mais il est indisperisable que les agriculteurs, comprenant qu'ils seront les principaux bénéficiaires de l'électrification rurale, fassent à leur tour un effort et transforment l'agriculture en une véritable industrie de la terre.

Les résultats obtenus à l'étranger et exposés par les précédents confércnciers montrent bien que les efforts pour l'utilisation de l'énergie électrique dans les travaux agricoles sont largement récompensés.

Le Génie Civil.

\section{L'électrification rurale en Grande-Bretagne}

L'électrification rurale a fait de grands progrès, ces demières annies, en Angleterre. Pendant un certain temps, les distributeurs n'avaient pas considéré la clientèle rurale comme intéressante; mais l'expérience ayant prouvé que, si au début, la vie d'un réseau rural est difficile, ce réseau devient rapidement avantageux pour son exploitant, les distributeurs anglais ont développé l'étendue de leurs réscaux ruraux.

L'auteur, qui possède en Angleterre une vaste propriété de 2.50 hectares, exploitée complètement à l'électricité, projette un film montrant les installations de cette exploitation.
Le courant est utilisé pour les divers travaux du labourage, de l'étable, de la laiteric, de la manutention des produits, pour le battage, le triage des grains, etc. Il sert mème dans les poulaillers et dans les ruches.

Les couveuses sont chauffées électriquement; les poulaillers sont éclairés et chauffés électriquement, ce qui a pour conséquence d'augmenter dans une très grande proportion la ponte des poules

Le chauffage et l'éclairage électrique des ruches procure également une forte augmentation du rendement du travail des abeilles. Le Génie Civil. 


\section{BIBLIOGRAPHIE}

Pour recharger ses aceus sur le secteur. -. "Comment construire soi-même le meilleur des redresseurs de courants alternatifs "(Le redresseur à collecteur tournant entrainé par moteur synchrone). - Par (. RIalland, in-16, broche, de to pages, avec 20 figures, Prix : 5 francs. Franco par la poste : $5 \mathrm{fr} .50$. - Editeurs : Desforges, Girardot et $C^{\circ}, 27$ et 29 , quai des Grands-Augustins, Paris (6"), ...- En vente a la Librairie ArthaulRey, Grande-Rue à Grenoble.

Avec les progrès de la 'T.S.F. le besoin se fait sentir pour lamateur électricien, d'un courant électrique constant et d'une intensité assez grande; il a alors recours aux accumulateurs et. bien souvent, disposant du courant du secteur, il désire les recharger lui-mème. Ce courant - ordinairement alternatif - ne permettant pas la recharge directe, lamateur trouvera dans ce petit ouvrage tous les éléments nécessaires pour construire un redresseur a collecteur tournant, silencieux et d'une marche sùre et régulière. Un chapitre sur la construction d'un transfo, amenant le courant au potentiel convenable pour charger la batterie, termine l'vuvrage.

S. V.

Marcellin Berthelot (1827-1907), par A. Boutaric, professeur à la Faculté des Sciences de Dijon. - Un volume in-8 de la collection d'letudes, de Documents et de Témoignages pour servir à l'histoire de notre temps. Editeur Payot. En vente à la librairie Arthaud-Rey, Grande-Rue, Grenoble. Prix : $15 \mathrm{fr}$.

Marcellin Berthelot, dont on va fêter le centenaire est, sans contredit, un des plus puissants génies qu ait vu éclore le $\mathrm{XIXe}$ siècle. Sa fécondité fut exceptionnelle. Son ouvre scientifique, qui ne comprend pas moins de trente volumes et de quinze cents notes ou mémoires, a porté sur les sujets les plus variés de la physique, de la chimie et des sciences de la vie. C'est lui qui, le premier, montra la possibilité de reproduire au laboratoire l'immense variété des corps qui s'élaborent au sein des êtres vivants et de créer de toute pièce des substances susceptibles de rivaliser, pour les applications, avec les produits naturels les plus précieux. La synthèse chimique, doù est sorti le prodigieux développement de la chimie moderne, est l'une de ses créations les plus originales. Mais dans combien d'autres domaines n'a-t-il pas montré toute sa puissance d'invention! L'étude de la chaleur dégagée dans les réactions ou thermochimie, les fermentations, les transformations si multiples et si variées qui se produisent au sein des végétaux, les explosifs, ont fait l'objet de découvertes dont une seule suffirait à immortaliser son nom. Par son amitié avec Renan, par ses relations avec les Taine, les Sainte-Beuve, les de Goncourt, les claude Bernard; il a été mêlé au mouvement intellectuel si intense en France à la fin du $X I X^{e}$ siècle.

Ce sont les divers aspects de cette pensée toujours en éveil, ouverte à tout ce qui est noble, grand et généreux, que M. Boutaric a essayé de fixer. Il nous expose en une langue simple, les grandes lignes d'une ceuvre scientificue que personne ne peut ignorer. En un chapitre curieux, il fait revive cette amitic d'un caractère si rare entre Berthelot et Renan.

(S. V.).

\section{$\star$}

Annuaire des forces hydrauliques (Wasserkraft Jahrbuch 1925-26), par le professeur K. Dantscher et Carl Reindl, ingénieur, Munick. - Un volume de 386 pages avec 186 figures et 7 tableaux. Richard Pflaum, éditeur, Munich.

Cette édition rédigée dans le même esprit que celle de l'Annuaire 1924 contient de nombreux articles signés de personnalités très compétentes sur les différents problèmes de la "houille blanche "qui se posent à l'heure actuelle. Divisé en quatre chapitres suivis d'un appendice, l'ouvrage relate les travaux les plus récents :

$1^{\circ}$ Dans le développement des installations hydrauliques en Autriche (Teigitschkraftwerk, Murkraftwerk, Spullerseewerk, Pernegg), en Suisse, en France, en Suede, en Norvège, en Italie (Reno-Limentra, Sila, Hône-Palerme) en Bulgarie en Yougoslavie en Roumanie en Grère en Russie et en Palestine :

$2^{\circ}$ Dans la mise en valeur des "forces hydrauliques " en particulier quant à leur application à l'industrie chimique et métallurgique. Successivement sont passées en revue la question d'équilibre des charges dans les grandes installations alpines par l'uti- lisation des lacs naturels ou de reservoirs artificiels ainsi que celle des stations de pompage qui clurant les périodes de hautes eaux emploiont l'excédent d'énergie à refouler dans le lac des eaux qui seront utilisées au moment des étiages;

$3^{\circ}$ Dans l'amenagement des "forces hydraulicues "sans nuire à la beauté des sites, remarques ayant leur importance et qui permettent de concilier an mieux dans les projets de nouvelles installations les points de vue technique, économique et artisticque. Des notes très intéressantes sur le barrage des vallées ainsi que sur la photographie en avion et son utilité dans les projets de cen trales completent ce chapitre :

40 Dians le calcul et la construction des turbines hydraulicues. Les domnées fondamentales nécessaires aux projets relatifs aux basses chutes posćes, le docteur V. Kaplan explique comment il fut concluit à créer la turbine qui porte son nom et qui a déja regu de nombreuses applications. I es quelques installations sucidoises et finlandaises décrites et très abondamment illustrés donnent une ide très nette de la superiorité des turbines modernes. Des graphiques et courbes de rendement precisent l'avantage de la turbine Kaplan par suite de la possibilité d'obtenir une marche economique dans de très grandes limites de charges.

Ce volume forme une documentation d'un réd intereat pour laquelle les auteurs n'ont pas craint d'unir la pratique à la theorie en faisant suivre les méthorles de calculs d'exemples numériques judicieusement choisis. Les différents sujets traités sont susceptibles de retenir l'attention des ingénieurs hydrauliciens et de tous les techniciens désireux de se tenir au courant des tendances en construction hydrotechnique. Nul doute que cette secomb' édition ne rencontre auprès des spécialistes la faveur gu'elle mérite.

A. $\%$.

Transformateurs de puissance, par Mared Mathieu. Un vol. $12 \% \times 19 \mathrm{c}, 500$ pages. Prix : $18 \mathrm{fr}$. I.ibrairie scientificule Albert Blanchard.

Ians cet ouvrage, element d'une encyclopédie électrotechnique, l'auteur a voulu surtout être utile. Dans ce but. il a cherche la nouveauté et la précision. Sans vouloir repéter les théories générales que l'on trouve dans tous les cours d'ilectrotechnique, l'auteur a cherché à présenter le sujet dans sa réalité industrielle.

L'ouvrage se divise en deux parties : dans la première le lecteur trouvera des données pratiques, des regles de construction indispensables, des chiffres précis applicables aux trinsformateurs de puissance.

La seconde partie est consacrée au calcul des bobines d'inductance, elle forme un recueil de formules établies par d'éminents physiciens et contient en outre des methodes de calcul de l'inductance des bobinages.

Edité avec soin, contenant des schémas clairs et de nombreux tableaux cet ouvrage aura sa place dans toutes les bibliothèques industrielles.

Mesures électriques, par Jean Granier. Un vol. 200 pages $11 \% \times 17 \%$. Prix broché : $9 \mathrm{fr}$. Collection Armand Colin.

M. Granier, préoceupé de faire un livre pratique, au courant des dernières méthodes, a délibérément proscrit de son exposé les appareils désuets qui n'ont plus qu'un intérêt historique; par contre, il insiste sur les montages modernes dont il discute les avantages ou les inconvenients, en ayant soin d'indiçuer l'approximation cue peut domner chaque type d'appareil.

Pour chaque quantité à mesurer, le livre indique un grand nombre de méthodes reposant sur des principes différents. De sorte que le lecteur, même s'il ne clispose que d'un matériel restreint, est toujours sùr de trouver exposé dans le livre le montage qui lui convient et la moilleure maniere de le mettre en ouvre. De nombreuses figures éclairent le texte.

Regrettons simplement que l'auteur n'ait pas cru devoir rappeler les noms des principaux savants ou ingénieurs auteurs des méthodes qu'il indique.

Pour ne citer qu'un exemple il est dommage que les jeunes générations ne voient pas le nom de M. Blondel associé à l'oscillographe et celui d'Hospitalier a l'ondographe et bien d'autres encore.

Le Gerant: P. LEGENDRB

Anc. Etabes Logendre, 14, rue Bellecordiere, Lyon. - J. Bataillard, Directeur 